\title{
Hidden Phase-type Markov Model for the Prediction of Onset of Farrowing for Loose-Housed Sows
}

\author{
Aparna U., ${ }^{\mathrm{a}, *}$, Lene Juul Pedersen ${ }^{\mathrm{a}}$, Erik Jørgensen ${ }^{\mathrm{a}}$ \\ ${ }^{a}$ Aarhus University, Department of Animal Health Science, P.O. Box 50, 8830-Tjele, Denmark
}

\begin{abstract}
High piglet mortality is an issue in the pig production. Evidence indicates that if the time of farrowing can be predicted, the mortality can be reduced through planned supervision or improved climate regulation. The aim of the study was to improve the prediction of onset of farrowing by monitoring pre-parturient behaviour of sows using several sensors and by developing an automated system for the prediction of time to farrowing. The resulting prediction model, named as Hidden Phase-type Markov Model (HPMM), assumes that a sow passes through the behavioural states Before Nest-Building, NestBuilding and Resting before reaching the Farrowing state. Each state was further split into phases, to allow a more realistic distribution of sojourn times. As these phases and states are unobservable, HPMM was used to calculate the probability of a sow being in given phase using the automatic sensor measures. Thus time to farrowing could be predicted at each time point. The prediction algorithm was validated on a sensor data set for about 35 sows, each followed from day 105 (day-105) since mating until the farrowing. Sensors include sow activity measured by video recording as well as by a photo-cell grid, and water consumption. The algorithm was evaluated using heuristic warning strategies e.g. that a warning should be generated when the expected time to farrowing was less than 12 hours (inspired by the regulation of floor heating systems). The performance of the sensors was evaluated. Different combinations of sensor types outperformed the use of a single sensor type. Using a combination of water and activity sensors the prediction algorithm gave a coherent warning period prior to farrowing (true warning) in $97 \%$ of the cases. The duration from start of the warning period to farrowing had a mean $11.5(\mathrm{SD}=4.6)$ hours. False warning periods ending before farrowing lasted on average only 0.7 hours per sow. The use of HPMM thus allowed a direct prediction of the time to farrowing, handling more than one sensor and a compact representation of historical sensor information.
\end{abstract}

Keywords: Hidden Markov Model, Phase-type distribution, prediction of onset of farrowing, sensors, automated system, Precision livestock

\section{Introduction}

Piglet mortality is one of the major causes for economic loss in pig production. An average of $13.7 \%$ of the live born piglets died before weaning in Danish sow herds in 2012 according to Vinther (2013). Although the variation between the Danish herds is not well documented, a Norwegian study (Andersen et al., 2007) has documented the mortality rates ranging from 5 to $24 \%$ and a Swedish study (Wallgren,

\footnotetext{
*Corresponding author: Tel: +45 71319660

Email addresses: aparna . udupi@gmail . com (Aparna U.), Lene. JuulPedersen@agrsci . dk (Lene Juul Pedersen), Erik. Jorgensen@agrsci.dk (Erik Jørgensen)

Please cite this article in press as: Aparna. U., et al. Hidden phase-type Markov model for the prediction of onset of farrowing for loose-housed sows. Compute. Electron. Agric. (2014), http://dx.doi.org/10.1016/j.compag.2014.07.008
} 
2013) has also discussed the variation between the herds and herd management. Baxter et al. (2011) reviews different studies in this field. The large variability between herds suggests a management component to the mortality, and several studies indicate that it is possible to reduce this mortality, especially in the herds with high mortality, either by increasing the supervision of the farrowings (White et al., 1996; Andersen et al., 2009), or through improved climate regulation during farrowing and the following days (Malmkvist et al., 2006). However, management efforts are only efficient if the required time can be minimized, and this requires that the time of farrowing can be predicted fairly precisely, particularly in large herds where the management effort per animal is often reduced. Based on mating time, the time of farrowing can be predicted within approximately \pm 2 days and this value is used to a large extent in farm planning. To obtain a better prediction of the time, it is necessary to include observations of the sows prior to farrowing.

Early studies have indicated that it is possible to base predictions on automatically recorded sensor data. These prior studies suggest that the change in the sow behaviour is reflected in change in the pattern of the sensor measurements. Erez and Hartsock (1990) described a system based on photo-cells to monitor periparturient activity of sows, and the experiment described by Bressers et al. (1994) showed significant changes in the ear base temperature around farrowing. The temperature increase started between 6-12 hours before farrowing.

Since these studies, a range of other sensors have become available. Thus a management tool for farrowing prediction can now use the online sensor information including feeding pattern, water intake, temperature or humidity in the pen level, and activity of the animal. For example, Oliviero et al. (2008) have used movement sensors (photocells and a thin-film ferroelectret force sensors) to detect the onset of farrowing in the crates.

The wide range of sensor technology has helped to record a huge amount of data; as a result statistical algorithms are necessary to extract behavioural patterns and combine measurements from multiple sensors into a useful information. Recently several studies have focused on statistical methods for handling data from online measurements. Different techniques have been used to extract the patterns, primarily different versions of the Kalman filter or Dynamic Linear Models (West and Harrison, 1997).

Madsen and Kristensen (2005); Madsen et al. (2005) looked at Dynamic Linear models for monitoring the health condition of young pigs by their drinking behaviour with an emphasis on diurnal drinking pattern. In this study a CUSUM approach based on the V-mask was used for detecting changes in the drinking pattern. Cornou and Lundbye-Christensen (2012) developed two methods to detect the onset of farrowing by monitoring the activity of the sow in the farrowing pen: 1. logistic dynamic generalized linear models for diurnal variation, and 2. modelling of activity using a cumulative sum based on daily variation. The warning signal for onset of farrowing, in either methods of Cornou and LundbyeChristensen (2012), is based on the detection of change in the activity pattern. The studies show that these changing pattern occurs mostly when the sow starts nest building (Baxter, 1984; Oliviero et al., 2008). However, most managemental tasks such as climate regulation require a direct estimate of time to farrowing. In such cases, the warning signal of Cornou and Lundbye-Christensen (2012) requires additional information about the distribution of the time from change point detection to start of farrowing.

Another promising class of models is that used in the analysis of time to failure. These models have so far not been implemented in farrowing prediction. Dayanik and Goulding (2009) gave a framework of detection of the distribution of an unobservable disorder time due to an unobservable cause. This type of model has only been applied in very few cases within livestock production. One such method is to use the Phase-type (PH) distribution for the event time to failure (Cox, 1955; Neuts, 1975) or in farrowing con- 
text, time to farrowing. PH-distributions are a special type of a Markov models in which the time spent in a stochastic process is modeled with phases through which objects in the model progress until the process is absorbed. Thus, in the prediction of farrowing, we would assume that the sow passes through different phases and is absorbed at farrowing. These behavioural phases are unobservable or hidden. However, if the sensor observations recorded on the pen level depend on the current phase of the sow, Hidden Markov Models (HMM) may be used to identify the latent phase. Such a combination with a PH-distribution furthermore gives an easy mechanism for aggregating and storing the information in historical registrations, and can easily handle observations from different types of sensors.

The purpose of this paper is to present and validate a prediction algorithm developed based on the above principles, and to validate the algorithm by applying simple heuristic warning strategies based on the results from the algorithm. The evaluation will compare different combinations of sensor types as input. The strategies include using the expected time to farrowing and the probability of farrowing. The farrowing prediction algorithm is planned to be a part of a farm management information system. The system will automatically collect sensor data and do the necessary calculations to make real-time predictions of farrowings. The real-time part of the algorithm will consist of a continuous revision of the probability distribution over the phases of the HMM based on sensor and farmer observations.

The predictions are based on herd specific parameters describing the distribution of the duration of each state of the farrowing process, as well as the conditional distribution of the sensor observations. The estimation of these parameters is described in chapter 3 of Aparna (2013).

\section{Materials and Methods}

In this section we will present the biological knowledge and principles used in the formulation of the algorithm, comprising the experimental setup and the different sensors used.

\subsection{Experimental data}

The data used in this study were collected from late 2008 to early 2009 in the experimental farm at the research center, Foulum, Denmark. 64 sows were introduced to the farrowing pen approximately seven days before expected farrowing. The sows were fed twice a day, 8:00 - 8:45 and 15:00 - 15:30, using an automatic feeding system. Management of the pen was restricted to a two hours period between 8:45 and 10:00, after the first feeding, where the pens were cleaned and $1 \mathrm{~kg}$ straw was provided daily on the floor.

Each farrowing pen had a number of sensors installed such as water valve and photo-cells as shown in Figure 1. In addition, video-recordings of each pen were made from the time when the sow was introduced until after farrowing. Additional visual analysis of these recordings includes identifying the start of farrowing (time of birth of first piglet) as well as a time point when the sow was nest-building. The onset of nest-building was recorded by an experienced observer and was identified based on the criteria described in Malmkvist et al. (2006), as the first occurrence of at least five front leg pawings per hour or repeated carrying of straw, without being interrupted by resting periods longer than 2 hours. The actual time of onset farrowing was used to validate the algorithm. The observed nest building time was used to interpret "the spikes of" the prediction curve but not directly for quantifying the algorithm performance.

The different measurements used for the development of the algorithm are described in the following. The data from the sensors were recorded with different time intervals, ranging from seconds to minutes. However, for this paper we consider the data pooled over half an hour intervals. Therefore a maximum of 48 observations were observed per day per sow. The pattern of these observations were used in the specification of statistical models described later on. The water consumption, video-activity and gridactivity data were collected from 45, 64 and 45 sows, respectively. The sensor information collected 


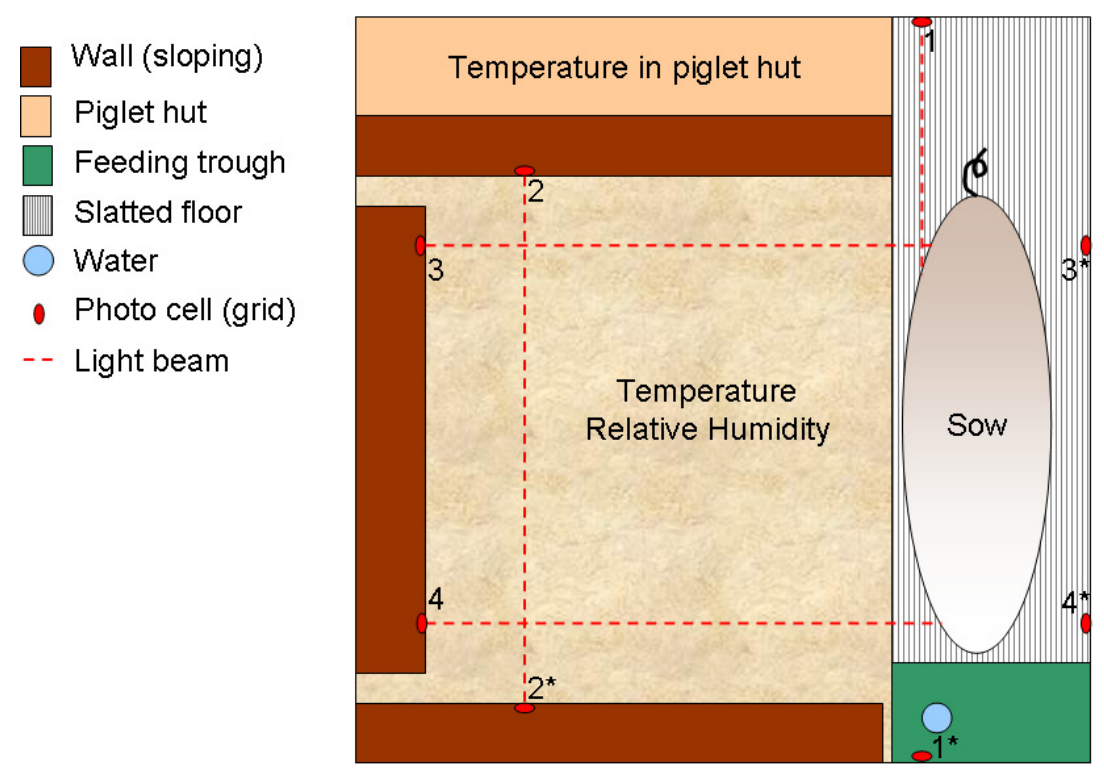

Figure 1: Sensor set up in the pen level.

before 105 days (day-105) after mating were excluded from the study. Some sows were discarded from the study because of failure of sensors. Furthermore, those sows for which data was recorded for less than 3 days before farrowing were also excluded from the prediction. The number of sows used in the study varied from 34 to 55. Sample size for different scenarios of sensor combination are presented together with the results.

Water Consumption of sows. The sensor for water consumption measures the water consumed by the sow as the number of rotations of the water valve (approx. $2 \mathrm{ml}$ of water per rotation). The water valve is situated on the food trough (see Figure 1). For the current study, the counts were summed up over half hour intervals. Preliminary data analysis suggested that log transformation of the water counts gave rise to residuals that better fulfilled the constant variance assumption compared to no-transformation and square root transformation. Therefore, we use the log-transformation of the water counts (denoted by $Y^{(w)}$ ) throughout the analysis. The pattern of water consumption is illustrated for one sow in Figure 2(a). The consumption pattern in sows was highly dependent on the time of the day. The sows consumed more water during feed intake and much less during the night. Furthermore, the water consumption occurred more frequently close to the farrowing, especially during the night. A disruption of the pattern was observed immediately prior to farrowing.

Activity of sows. Activity measures are based on the video recordings observations. The activity measure is the number of pixel changes from frame to frame in the video recordings. The preliminary studies indicated that, along with the mean of the activity over an interval it is realistic to consider the variation of the activities during the interval. Hence, in this paper, the sow activity was evaluated on two measures: mean (meanActivity) and the sample standard deviation (sdActivity) of the activity measures on half an hour interval. We use log-transformation of both the quantities throughout the model and they are denoted by $Y^{(A m)}$ and $Y^{(A s d)}$, respectively. To keep the model simple, we assume these two variables are conditionally independent. The patterns in the observations are illustrated in Figure 2(b) and Figure 2(c). The sows were less active during the night time as compared with the day time. The sdActivity was also high during the day time. However, sdActivity shows more deviations during the night time, probably because 
of the changing lying position. In addition to this, both meanActivity and sdActivity have increased prior to and during parturition.

Grid measurement. Grid measurements are the interruption of photocell measurements due to the movements of sow in the pen. The grid set-up consists of four pairs of emitter and a receiver, $\left\{\left(1,1^{*}\right),\left(2,2^{*}\right)\right.$, $\left(3,3^{*}\right)$ and $\left.\left(4,4^{*}\right)\right\}$ as shown in Figure 1 and were mounted at the height of $80-85 \mathrm{cms}$. If the sow is in the path of the beam, the beam is interrupted; for example, the receiver $1^{*}$ (also the receivers $3^{*}$ and $4^{*}$ ) in Figure 1 does not receive the beam from the emitter 1 ( 3 and 4 ) and hence the beam is interrupted by the sow in its path. Thus, the grid measures it as an activity in that direction, at that time. However, if the sow was in the same position in the previous time of recording, then it is not considered as an activity. Furthermore, if the movements take place below the lane of photo cells, for example, lying, sleeping, it will not be measured by the grids. In this paper, the total activity measured by the four cells were summed up over half hour intervals. We use the log-transformed activity measure which we denote $Y^{(g)}$. The sows were less active during the night time and comparatively more active during the herd management. Also the sows showed significant activities before the parturition and deviation in the night time pattern (Figure 2(d)).

Time variables. The data used for the prediction algorithm includes the date of mating. In addition, from the examples given in Figure 2, it is evident that there is a clear diurnal rhythm with reduced activity during night time, at least in the the first days after introduction to the pen. Therefore, it was necessary to include a continuous time variable to denote the time of the day between 0 and 24 hours in addition to the time since the mating or time to farrowing. We denote this variable by TOD to indicate time of the day and it takes the values $\zeta$ such that $\zeta \in[0,24)$. These values are used for generating the harmonic covariates while modelling the conditional distribution of sensors.

\subsection{Behavioural knowledge and Statistical methods used to formulate the Prediction Model}

This section describes the biological and behavioural background of a sow during the pre-parturition and parturition states which lead to the formulation of a stochastic model for the prediction of the farrowing. The current understanding of the physiological and behavioural changes during the pre-parturition is based both on study in semi-natural conditions and under production conditions. The current section also describes the statistical background of the prediction model.

Behavioural Background. The relevant period for the prediction of farrowing in the production system starts when the sow is mated. If she becomes pregnant she will farrow approximately 115 days after mating. At approximately day-105 after mating, the sow will be transferred into a farrowing pen or farrowing system (however, in the commercial farms the sows may be moved about day-110). The sensors will start recording the data as well as the automatic monitoring of the sow will start at this time. Thus, this is the interesting time period for the prediction algorithm.

From mating to 1-2 days prior to farrowing the sow will show a regular behavioural pattern with a clear diurnal rhythm. When the sows are being moved to the farrowing unit a temporary change in the behaviour may take place for 1-2 days due to a change of environment. As the time of farrowing approaches hormonal changes in the sows will motivate the sow to change behaviour and show nest building behaviour. The behavioural changes will typically be present during the last 24 hours before parturition (Baxter, 1984; Jensen, 1989; Wischner et al., 2009) and were expected to show up in the diurnal pattern in the sensor data. Nest building behaviour declines before farrowing, partly due to an elevation in oxytocin (Castrén et al., 1993).

Thus the pre-parturition behaviour of a sow can be broadly classified into three states, Before NestBuilding, Nest-Building and Resting, as illustrated in Figure 3. These three behavioural states of the sow 


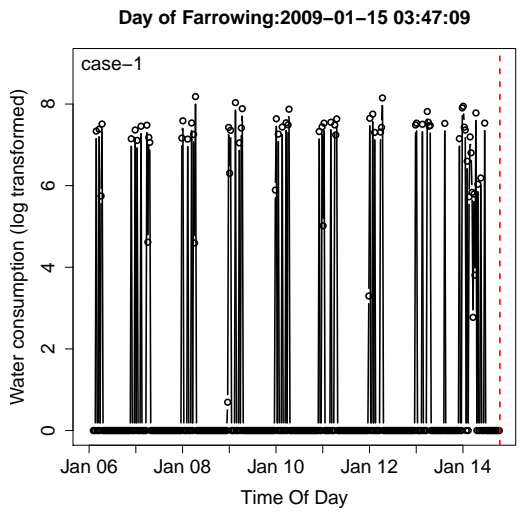

(a) water consumption

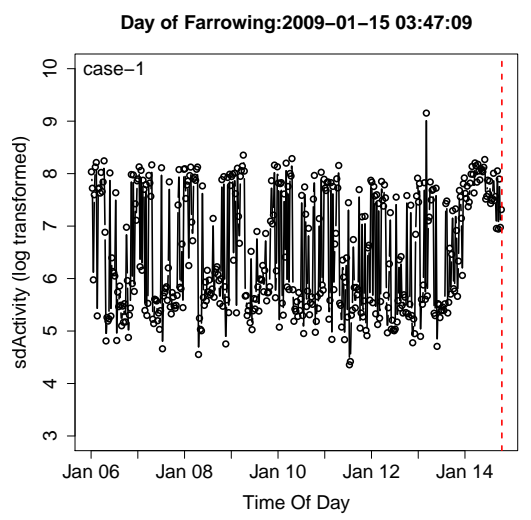

(c) sdActivity

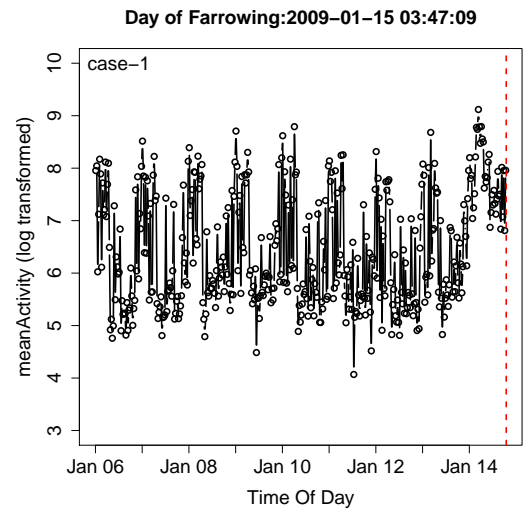

(b) meanActivity

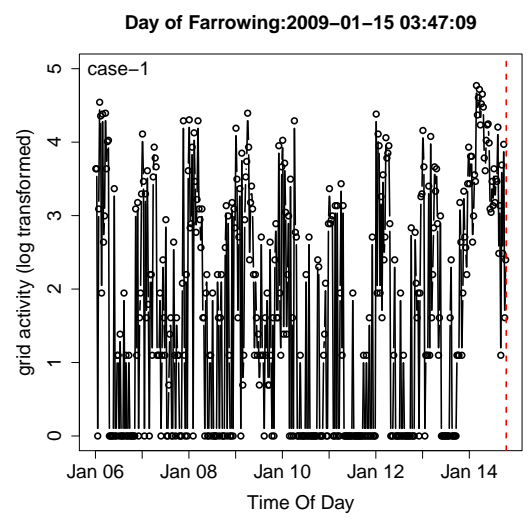

(d) grid-activity

Figure 2: Illustration of water consumption, meanActivity, sdActivity and grid-activity patterns for a sow, pooled over half hour intervals (on their log transformations). The dotted vertical line in the right indicates the actual time of onset of farrowing. 
can be seen using both behavioural and physiological measures. In addition, we have the Farrowing state, where the birth of the first piglet defines the beginning of the state. Thus, the Resting state could be the start of the parturition process. We use the term 'farrowing process' to refer the gestational progression and process.

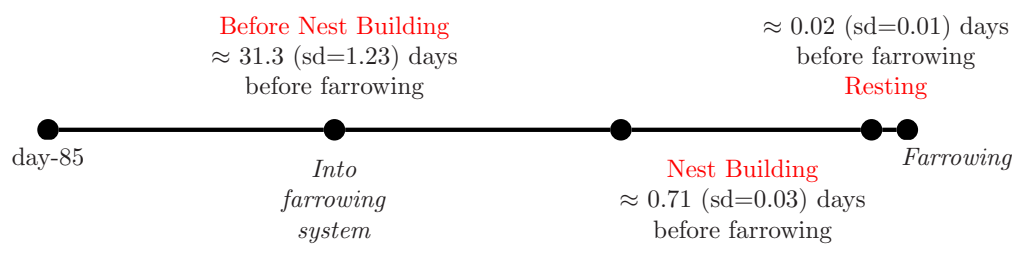

Figure 3: Pre-parturition Behavioural states of the sow (Not to scale).

The behavioural or motivational states are latent and not directly observable. It is well recognized between researchers that the states can be indirectly observed, by combining different behavioural and physiological observations (Thodberg et al., 2002; Malmkvist et al., 2006; Wischner et al., 2009), although a direct quantitative model has not been formulated. However, the use of different sensors mounted in the pen level have made it easier to automatically record the data whose distributions are influenced by the latent states. Hence it has become realistic to formulate such a quantitative model.

Stochastic Model. HMM is a well established technique for modelling processes similar to the preparturition behaviour of the sow, for example, monitoring disease progression, where a system progresses through different states. We may observe other variables, and because the distribution of these variables are different for different states, we may use these observations to improve our knowledge about the state of the system at each time step. However, in the usual formulation, HMM assumes that the sojourn times of the latent states are exponentially distributed. This is clearly not the case for the pre-parturition states. The mean duration of each of these states varies from study to study, but is typically approximately 24 hours for the Nest-Building state and 6 hours for the Resting state and 115 days of total gestation period. The variability of these durations is not well documented except for the total gestation period with a standard deviation of approximately 2 days. The distribution of total gestation period is close to (left-truncated) normal. Prior studies e.g. Castrén et al. (1993); Malmkvist et al. (2012) indicate non-exponential sojourn time distribution of these states (mean is not equal to variance). Therefore, we approximate the sojourn time distributions of the behavioural states to Gamma distributions. Hence HMM can not be implemented directly for our purpose.

This was solved by splitting up the states into a number of smaller divisions called phases (as illustrated in Figure 4) each of whose sojourn times follows exponential distribution such that the total sojourn time distribution of each state is a Gamma or to be more specific an Erlang distribution, as the number of phases in each state is an integer value.(An Erlang distribution is a special case of a Gamma distribution with an integer valued scale parameter). And the total gestation period is the sum (or convolution) of these three Erlang distributions which is a PH-distribution absorbed at Farrowing. Therefore, farrowing process over phases is a Markov process and so, HMM was constructed over the pre-parturition phases instead of states. Thus, an event time to farrowing can be modelled using PH-distribution. By the theory, $\mathrm{PH}$-distribution is a probability distribution of a random variable of an absorbing Markov process.

The distribution of the corresponding sensor measurements were conditioned on the phases. Figure 5 shows an analogue of HMM. At time $t$, the sow will be at phase $U_{t}=u, u \in \mathbf{U}$ where $\mathbf{U}$ is 
the set of all pre-parturition phases. However, prediction starts only after the sow was introduced into the farrowing pen. An observation $Y_{t}$ was measured by a sensor from the distribution associated with the phase $u$. Such an observation can be subsequently used to update the probabilities over the phases. For simplicity, consecutive predictions were performed at an equal time interval of $\delta$ hours and the sensor observations were pooled to fit into the prediction time. Therefore, the time set for the prediction is $\mathcal{T}=\left\{t_{I}, t_{I}+\delta, \ldots, t_{I}+N \delta\right\}$ where $N$ is such that $t_{I}+N \delta \quad\left(\leq t_{F}\right)$ is the time of last observation before farrowing. Since the time of farrowing $\left(t_{F}\right)$ is unknown in reality, $N$ or $t_{I}+N \delta$ is also unknown.

Furthermore, The PH-distribution can use the updated phase probabilities to compute the expected time to onset of farrowing at the time of prediction. The proposed farrowing prediction model is a combination of HMM and PH-distribution and therefore, the model is named as Hidden Phase-type Markov Model (HPMM).

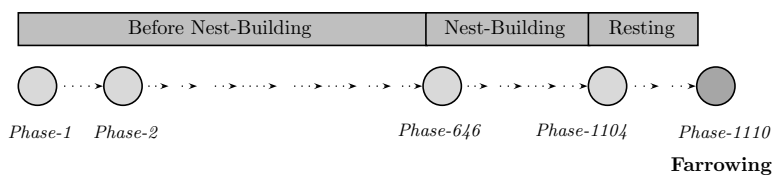

Figure 4: An illustration of Markov process with the states divided into phases. The phases, Phase-1, Phase-646 and Phase-1104 are the first phase of the states Before Nest-Building, Nest-Building and Resting. Phase-1110 is the absorption phase or the first phase of Farrowing.

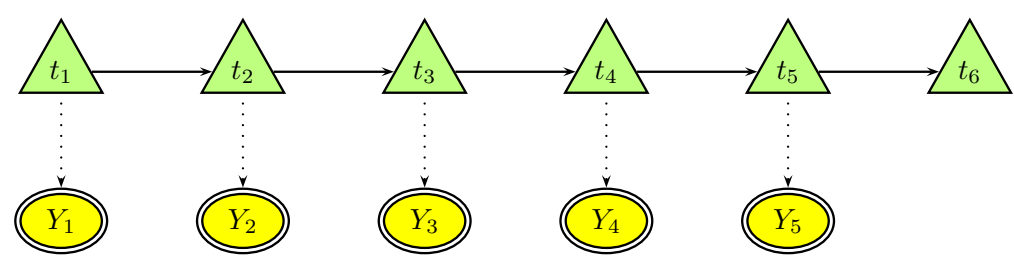

Figure 5: An analogue of the Hidden Markov process (HMM). The sequence $\{t\}$ denotes time instance at which an observation $Y_{t}$ are observed. At time $t$ the system stays in phase $U_{t}=u$ which is unobservable. However, $Y_{t}$ 's are observed from the distribution associated with the phase $u$. $Y_{t}$ could be any measure such as water consumption, activity, temperature etc.

Warning Strategies for validating the algorithm. The purpose of the prediction was to be able to send out warning signals that may, for example, activate the heating equipment or alert the farmer for the special attention. The algorithm we present in this article calculates the phase probabilities using all the available information at each time step, and can calculate the necessary statistics to predict the onset of farrowing. We now need a warning strategy to tell when to send out the warnings. A warning strategy is defined as a mapping between a probability distribution for time to farrowing at a given time and a decision to either send a warning or do-nothing.

\subsection{Notations}

We denote the preparturition behavioural states Before Nest-Building, Nest-Building, Resting and Farrowing by $\mathcal{S}_{1}, \mathcal{S}_{2}, \mathcal{S}_{3}$ and $\mathcal{S}_{4}$ respectively. The number of phases in the $i^{\text {th }}$ state $\mathcal{S}_{i}$ is denoted by $m_{i}$, 
$i=1,2,3$. Therefore, there are $\mathbf{M}=\sum_{i=1}^{3} m_{i}$ transient phases. We assume only one phase in the Farrowing state and is denoted by $u_{F}$. The successive phases are denoted by an ordered sequence Phase-1, Phase- $2, \ldots$ or simply $1,2, \ldots$ such that Phase- $m_{1}$ is the last phase of state $\mathcal{S}_{1}$, Phase- $\left(m_{1}+1\right)$ and Phase$\left(m_{1}+m_{2}\right)$ are the first and last phases of state $\mathcal{S}_{2}$, Phase- $\left(m_{1}+m_{2}+1\right)$ and Phase- $\left(m_{1}+m_{2}+m_{3}\right)$ are the first and last phases of state $\mathcal{S}_{3}$ and Phase- $\left(m_{1}+m_{2}+m_{3}+1\right)=u_{F}$ is the farrowing phase.

Let $\mathbf{U}$ denote the set of all $\mathbf{M}$ phases and $\mathbf{U}_{i}$ denote the subset of $\mathbf{U}$ that consists all the phases of state $\mathcal{S}_{i}$, i.e. $\mathbf{U}_{1}, \mathbf{U}_{2}$ and $\mathbf{U}_{3}$ has $m_{1}, m_{2}$ and $m_{3}$ number of phases, respectively. Let $u \in \mathbf{U}_{i}$ denote any phase $u$ in $\mathbf{U}_{i}$. Further, $U_{t}$ is a random variable that denotes the phase of the sow at time $t$ and $U_{t}=u$ implies that the sow occupies phase $u$ at time $t$. The states are indexed by $i$ or $j$ and the phases by $u$ or $v$.

The vector of probabilities over the transient phases at time $t$ is denoted by $\boldsymbol{\alpha}_{t}$. Let $\left(\boldsymbol{\alpha}_{t}\right)_{\mathbf{U}_{i}}$ denote the elements of $\boldsymbol{\alpha}_{t}$ corresponding to all the phases in state $\mathcal{S}_{i}$. The vector of probabilities of the transient phases corresponding to the time when the farrowing process begins is denoted by $\boldsymbol{\alpha}_{0}$. The probability corresponding to the farrowing phase is denoted by $(\alpha)_{F}$.

Phase-Type Distribution. Consider an $(\mathbf{M}+1)$-phase and continuous-parameter Markov process with $\{1,2, \ldots, \mathbf{M}\}, \mathbf{M} \geq 1$, transient phases and an absorption phase $(\mathbf{M}+1)$, with rate matrix,

$$
\mathbf{Q}=\left(\begin{array}{cc}
\mathbf{S} & \mathbf{S}^{\mathbf{0}} \\
\mathbf{0} & 0
\end{array}\right)
$$

where $\mathbf{S}_{\mathbf{M} \times \mathbf{M}}$, corresponds to the transient phases; $\mathbf{S}^{\mathbf{0}}{ }_{\mathbf{M} \times 1}$, corresponds to the absorbing phase from the transient phases. In our case, $\mathbf{S}_{\mathbf{M} \times \mathbf{M}}$ is sparse and will only have non-zero values at the diagonal and super-diagonal.

Let $\left(\boldsymbol{\alpha}, \alpha_{\mathbf{M}+1}\right)$ be the row-vector of initial phase probabilities with $\boldsymbol{\alpha}$ corresponding to the transient phases and $\alpha_{\mathbf{M}+1}$ corresponding to the absorption phase.

Then, the probability distribution of the time until absorption in the Markov chain $\mathbf{Q}$, on $(0, \infty)$ is,

$$
\operatorname{Pr}(T \leq \delta)=1-\boldsymbol{\alpha} \mathbf{e}^{\delta \mathbf{S}} \mathbf{1}, \quad \delta \in \mathcal{R}^{+} .
$$

This is a Phase-type distribution (PH-distribution) (Neuts, 1978) and is represented by a pair ( $\boldsymbol{\alpha}, \mathbf{S})$. Here, $T$ denotes the event time to absorption and $\mathbf{1}$ is the unit column-vector and $\mathbf{e}$ is the matrix exponential (Bernstein, 2009).

For the farrowing process, the absorbing phase is at Farrowing and therefore, $\alpha_{\mathbf{M}+1}=(\alpha)_{F}=$ $1-\boldsymbol{\alpha} 1$.

The transition probability matrix for the time interval $\delta$ is given by, (Asmussen et al., 1996),

$$
\mathbf{P}_{\delta}=\mathbf{e}^{\mathbf{S} \delta} .
$$

We use this formula for updating the phase probabilities in each time step.

The moments of the distribution are given by

$$
\mathbb{E}\left[T^{n}\right]=(-1)^{n} n ! \boldsymbol{\alpha} \mathbf{S}^{-n} \mathbf{1} \quad n=1,2,3, \ldots
$$

Therefore, the mean of the PH-distribution is

$$
\mathbb{E}[T]=-\boldsymbol{\alpha} \mathbf{S}^{-1} \mathbf{1}
$$


and the variance can be found from the second moment,

$$
\mathbb{E}\left[T^{2}\right]=2 \boldsymbol{\alpha} \mathbf{S}^{-2} \mathbf{1} .
$$

Thus we have the basis for calculating the input to our warning algorithm. Other notations are explained as and when they are introduced.

\section{Prediction Algorithm}

The following are the steps of the algorithm for the prediction of onset of farrowing for an individual sow.

\subsection{Initializing the algorithm for a sow}

In principle, the farrowing process starts on the day the sow was successfully mated. Thus we know that the sow is in the first phase. Initial evaluation studies showed that the distribution of sojourn time from mating to farrowing (gestation length) could be described adequately with a constant part of 85 days and a gamma distributed final part. This approximation lead to a markedly lower number of phases. Therefore, to avoid too many phases, the model for the first part of the gestation period is slightly modified. Therefore, the model was reformulated, such that all the sows were entered into Phase- 1 on day- 85 after mating, Thus, the phase probabilities on the day 85 were defined as the vector, $\boldsymbol{\alpha}_{0}$ with the first element 1 and the rest 0 s. If $\delta_{I}$ is the time interval between day- 85 after mating and the day the sow was introduced into the farrowing system, the transition matrix, $\mathbf{P}_{\delta_{I}}$ was found from (2) and the phase probabilities of the sow on the day of insertion were then calculated as,

$$
\boldsymbol{\alpha}_{t_{I}}^{(0)}=\boldsymbol{\alpha}_{0} \mathbf{P}_{\delta_{I}}
$$

where $t_{I}$ is the time that the sow was introduced into the farrowing system. We also assume that the sensors mounted in the pen starts recording the data from time $t_{I}$.

\subsection{Half-hourly updating for a sow}

As mentioned in sec. 2.1, the observations were pooled and measured at the fixed time interval $\delta$. Therefore, the phase transitions of the sow were calculated on every interval $\delta$ after $t_{I}$, where $\delta=0.5$ hours in this paper. If $t$ is the current time then the phase transition of the sow at time $t+\delta$ is predicted as

$$
\boldsymbol{\alpha}_{t+\delta}^{(0)}=\boldsymbol{\alpha}_{t} \mathbf{P}_{\delta} .
$$

Here, the transition matrix, $\mathbf{P}_{\delta}$, is again found using (2). As the time interval $\delta$ is constant, we only need to calculate $\mathbf{P}_{\delta}$ once.

Furthermore, as mentioned the HMM technique allows us to use any sensor observation, measured at time $t$, to revise the phase probabilities of time $t+\delta$. Since we assume that the sensor measures are conditionally independent given the phase, the phase probabilities were revised for each sensor measure available at the given time. The conditional independence also means that the sequence of sensors in the revision is irrelevant and any missing sensor measure does not influence the revision of probabilities. Let there be $N_{s}$ number of sensors available at time $t$ (note that the time indicator is omitted in the notation $\left.N_{s}\right)$. Let $Y_{t}^{\left(n_{s}\right)}$ be the observation measured at time $t$ by $n_{s}{ }^{t h}$ sensor. Then, the phase probabilities given the $n_{s}$ number of sensors is,

$$
\boldsymbol{\alpha}_{t+\delta}^{\left(n_{s}\right)}=\frac{\boldsymbol{\alpha}_{t+\delta}^{\left(n_{s}-1\right)} \cdot \operatorname{Pr}\left(Y_{t}^{\left(n_{s}\right)} \mid U\right)}{\operatorname{Pr}\left(Y_{t}^{\left(n_{s}\right)}\right)}, \quad n_{s}=1,2, \ldots, N_{s}
$$


where $\operatorname{Pr}\left(Y_{t}^{\left(n_{s}\right)} \mid U\right)$ is the row vector of probabilities of $\left(Y_{t}^{\left(n_{s}\right)} \mid\right.$ Phase-1), $\left(Y_{t}^{\left(n_{s}\right)} \mid\right.$ Phase-2),... $\left(Y_{t}^{\left(n_{s}\right)} \mid\right.$ Phase-M) and $\operatorname{Pr}\left(Y_{t}^{\left(n_{s}\right)}\right)=\boldsymbol{\alpha}_{t+\delta}^{\left(n_{s}-1\right)} \operatorname{Pr}\left(Y_{t}^{\left(n_{s}\right)} \mid U\right)^{\top}$. Here, $A^{\top}$ denotes the transpose of $A$ and $A \cdot B$ is the element-wise multiplication of $A$ and $B$.

In the current paper, the phase probabilities were updated for the sensor measures $Y_{t}^{\left(n_{s}\right)} \in\left\{Y_{t}^{(w)}, Y_{t}^{(A m)}, Y_{t}^{(A s d)}, Y_{t}^{(g)}\right\}$ and are discussed in sec. 3.3. Therefore, $\boldsymbol{\alpha}_{t}^{\left(n_{s}\right)} \in\left\{\boldsymbol{\alpha}_{t}^{(A m)}, \boldsymbol{\alpha}_{t}^{(A s d)}, \boldsymbol{\alpha}_{t}^{(g)}, \boldsymbol{\alpha}_{t}^{(w)}\right\}$ for $n_{s}=1, \ldots, N_{s}$.

The vector of final phase probabilities predicted for time $t+\delta$ is, $\boldsymbol{\alpha}_{t+\delta}=\boldsymbol{\alpha}_{t+\delta}^{\left(N_{s}\right)}$ if $N_{s}$ sensor information is available; otherwise, $\boldsymbol{\alpha}_{t+\delta}=\boldsymbol{\alpha}_{t+\delta}^{(0)}$.

Therefore, the probability of farrowing phase is,

$$
\left(\alpha_{t+\delta}\right)_{F}=1-\boldsymbol{\alpha}_{t+\delta} \mathbf{1} .
$$

The phase transition with time and the revision of the phase probabilities using the available sensor observations were continued alternatively for time $t=t_{I}, t_{I}+\delta, t_{I}+2 \delta, \ldots, t_{I}+N \delta$. At the end of each time step, before considering the next half-hourly updating of phase transition (as in (6)), the necessary statistics for raising the warning were calculated. Sec. 4.2 discusses some of such statistics used in this study.

The algorithm will proceed until farrowing has been observed by the farmer, and not directly by the sensor observations.

\subsection{Updating $\boldsymbol{\alpha}_{t}$ Using the Sensor Information}

The prediction algorithm was built and implemented to use the sensor information such as water observations, video-activity and grid-activity measures. As described in sec. 2.2, the prediction process starts from the day the sow was introduced into the farrowing system. At time $t$, if any evidence from the sensors is available, it was used to update the phase probabilities $\boldsymbol{\alpha}_{t+\delta}$ as follows:

We assume that at a given time $t$, the conditional distribution of meanActivity, sdActivity and gridactivity are distributed as follows,

$$
\begin{aligned}
\left(Y_{t}^{(A m)} \mid u \in \mathbf{U}_{i}\right) & \sim \mathcal{N}\left(\mu_{i \zeta}^{(A m)}, \sigma_{i}^{2(A m)}\right) \\
\left(Y_{t}^{(A s d)} \mid u \in \mathbf{U}_{i}\right) & \sim \mathcal{N}\left(\mu_{i \zeta}^{(A s d)}, \sigma_{i}^{2(A s d)}\right) \\
\left(Y_{t}^{(g)} \mid u \in \mathbf{U}_{i}\right) & \sim \mathcal{N}\left(\mu_{i \zeta}^{(g)}, \sigma_{i}^{2(g)}\right)
\end{aligned}
$$

where $\mu_{i \zeta}^{(A m)}, \mu_{i \zeta}^{(A s d)}$ and $\mu_{i \zeta}^{(g)}$ are the mean level of meanActivity, sdActivity and grid-activity measures respectively for the state number $i=1,2,3$ and time of the day $\zeta \in[0,24)$. We also assume that ${\sigma_{i}^{2}}^{(Y)}=\sigma^{2^{(Y)}}$, for state number $i=1,2,3$ and $Y \in\{$ meanActivity, sdActivity, grid-activity $\}$.

These density functions were used in the expression (7) as the likelihood of $\left(Y_{t}^{\left(n_{s}\right)} \mid U\right)$ and the phase probabilities $\boldsymbol{\alpha}_{t+\delta}^{(A m)}, \boldsymbol{\alpha}_{t+\delta}^{(A s d)}$ and $\boldsymbol{\alpha}_{t+\delta}^{(g)}$ corresponding to meanActivity, sdActivity and grid-activity, respectively, were calculated.

The water observation measured at time $t$ is assumed to be from a mixture distribution for the given state; the mixing is over a number of components. These components can be seen as different types of drinking behaviour that the sow may select. Furthermore, the mixing proportions were associated with the time of measurement. For the simplicity, we assume that the consumption pattern of one state is 
independent of other states and, within the state, the water consumption in each component follows a distribution independent of the other component. The probability of observing the water consumption in $k^{\text {th }}$ component $\mathcal{C}_{k}^{(i)}$ of state $\mathcal{S}_{i}, \quad i=1,2,3$ is defined as

$$
\pi_{k}^{(i)}=\operatorname{Pr}\left(\mathcal{C}_{k}^{(i)} \mid \mathcal{S}_{i}\right), \quad k=1, \ldots, K^{(i)}
$$

where $K^{(i)}$ is the number of components in state $\mathcal{S}_{i}$.

Therefore, the density of observing $Y_{t}^{(w)}$ at time $t$ as the $k^{t h}$ component of the $i^{t h}$ state is

$$
\left(Y_{t}^{(w)} \mid \mathcal{C}_{k}^{(i)}, \mathcal{S}_{i}\right) \sim \mathcal{N}\left(\mu_{i \zeta}^{(w)}{ }^{(k)}, \sigma_{i}^{2^{(w)}}{ }^{(k)}\right)
$$

Furthermore, we assume that $\left(Y_{t}^{(w)} \mid \mathcal{C}_{k}^{(4)}, \mathcal{S}_{4}\right)=\left(Y_{t}^{(w)} \mid \mathcal{C}_{k}^{(3)}, \mathcal{S}_{3}\right)$. Therefore, the likelihood of $Y_{t}^{(w)}$ given $\mathcal{S}_{i}$ is

$$
\operatorname{Pr}\left(Y_{t}^{(w)} \mid \mathcal{S}_{i}\right)=\operatorname{Pr}\left(Y_{t}^{(w)} \mid \mathcal{C}_{k}^{(i)}, \mathcal{S}_{i}\right) \quad \operatorname{Pr}\left(\mathcal{C}_{k}^{(i)} \mid \mathcal{S}_{i}\right)_{t} \quad i=1,2,3,4
$$

Hence, by Bayes' theorem, the posterior of each state given the observation is

$$
\operatorname{Pr}\left(\mathcal{S}_{i} \mid Y_{t}^{(w)}\right)=\frac{\operatorname{Pr}\left(Y_{t}^{(w)} \mid \mathcal{S}_{i}\right) \operatorname{Pr}\left(\mathcal{S}_{i}\right)_{t}}{\sum_{j=1}^{4} \operatorname{Pr}\left(Y_{t}^{(w)} \mid \mathcal{S}_{j}\right) \operatorname{Pr}\left(\mathcal{S}_{j}\right)_{t}} ; \quad i=1,2,3,4
$$

where $\operatorname{Pr}\left(\mathcal{S}_{i}\right)_{t}=\left(\boldsymbol{\alpha}_{t}\right)_{\mathbf{U}_{i}} \mathbf{1}_{m_{i}}$ is the sum of $\boldsymbol{\alpha}_{t}$ elements corresponding to state $\mathcal{S}_{i}, i=1,2,3$, $\operatorname{Pr}\left(\mathcal{S}_{4}\right)_{t}=\left(\alpha_{t}\right)_{F}$ and $\mathbf{1}_{m_{i}}$ is the unit vector of size $m_{i}$.

Hence, the phase probabilities were updated as

$$
\left(\boldsymbol{\alpha}_{t}^{(w)}\right)_{\mathbf{U}_{i}}=\operatorname{Pr}\left(\mathcal{S}_{i} \mid Y_{t}^{(w)}\right) \frac{\left(\boldsymbol{\alpha}_{t}\right)_{\mathbf{U}_{i}}}{\operatorname{Pr}\left(\mathcal{S}_{i}\right)} ; \quad i=1,2,3
$$

where $\left(\boldsymbol{\alpha}_{t}^{(w)}\right)_{\mathbf{U}_{i}}$ are the elements of $\boldsymbol{\alpha}$ corresponding to the phases in state $\mathcal{S}_{i}$; similarly the $\left(\boldsymbol{\alpha}_{t+\delta}^{(w)}\right)_{\mathbf{U}_{i}}$.

\section{Computational and validation plan}

The prediction algorithm described above is tested using the experimental data described in sec. 2.1. For the validation of the algorithm, in the current paper, we use the same sow and sensor data as used for estimating the model parameters described in chapter 3 of Aparna (2013). For each sow, the sensor data were used sequentially, thus pretending that only the data from time of insertion up to the half hourly time of prediction was available at each prediction. If data from a sensor were missing at a given time the corresponding updating of the phase probabilities was omitted. Furthermore, if all the sensor observations were missing at the time of prediction, the algorithm handles it by calculating only the phase transition over time. The validation could have been made using a cross-validation approach where the observations from each sow was evaluated with parameters estimated from data for other sows. See e.g. Chapter 5 of James et al. (2013) for other possibilities. However, because of the sample size it was expected that each sow had so little influence on the parameter estimates that the large extra computing time for the cross-validation was not worth the effort.

\subsection{Parameters and values}

The model parameters were estimated by an EM-algorithm inspired by the Baum-Welch algorithm by updating Forward-Backward probabilities as described in detail in chapter 3 of Aparna (2013). We refer to the thesis for more information. However, as the estimated parameters were used for the prediction algorithm they are briefly described in the following. 


\subsubsection{Sojourn Time Distribution, Number of Phases and Transition Rates}

The sojourn times of the pre-parturition behavioural states were assumed to be Gamma distributed. The mean and variance for these states are given in Table 1. The values can be interpreted as follows: on average, a sow spends 17.02 hours ( $\mathrm{SD}=0.80$ hours) in the Nest-Building state; whereas the sojourn time for Resting, with the limited information, was estimated to be 0.53 hours ( $\mathrm{SD}=0.22$ hours) before farrowing. These values are in good agreement with published values for nest-building times, although in the low range. If the sojourn times were exponentially distributed, the SD would be equal to the mean. The low SD thus support the use of the PH-distribution. The total duration is approximately 32 days after day-85 or 117 days after mating.

Hence, the number of phases and the transition rates of a state are the parameters of the corresponding Erlang distribution. They are calculated to be $\left(m_{1}, m_{2}, m_{3}\right)=(645,458,6)$, so that the sow passes through 1109 phases before the farrowing and $1110^{\text {th }}$ phase is the beginning of Farrowing. The process exits at the rate $\lambda_{1}=0.86$ transitions per hour between the successive phases within the Before NestBuilding state and enters into the first phase of Nest Building state from the last phase of Before NestBuilding. Further, it transits between the successive phases of Nest-Building state with the rate $\lambda_{2}=$ 26.91 transitions per hour and enters into Resting state. The process will transit between the successive phases in the Resting state with the rate $\lambda_{3}=11.4$ transitions per hour and then enters into the Farrowing state.

Table 1: Mean duration (with their SD) of stay and number of phases in each state for a sow along with within state and between states transition rates.

\begin{tabular}{|c|c|c|c|c|}
\hline \multirow{2}{*}{ State } & \multicolumn{2}{|c|}{ Duration (hours) } & \multirow{2}{*}{$\begin{array}{l}\text { No. of } \\
\text { Phases }\end{array}$} & \multirow{2}{*}{$\begin{array}{r}\text { Transition rate } \\
\text { (per hour) }\end{array}$} \\
\hline & Mean & SD & & \\
\hline Before Nest-Building & 751.20 & 29.58 & 645 & 0.86 \\
\hline Nest-Building & 17.02 & 0.80 & 458 & 26.91 \\
\hline Resting & 0.53 & 0.22 & 6 & 11.40 \\
\hline
\end{tabular}

For these values, the phase transition probabilities were calculated using (2) by constructing the $\mathbf{S}$ matrix of PH-distribution. The $\mathbf{S}$ matrix can be divided into $3 \times 3$ sub-matrices corresponding to each pre-parturition state. The $i^{t h}$ sub-matrix on its diagonal is of size $m_{i} \times m_{i}$ such that, the values on the diagonal are $-\lambda_{i}$ and super-diagonal are $\lambda_{i}$; for $i=1,2,3$. These correspond to the phases of $i^{t h}$ state. For example, for $\left(m_{1}, m_{2}, m_{3}\right)=(2,3,2), \mathbf{S}$ is given by,

$$
\mathbf{S}=\left[\begin{array}{cc|ccc|cc}
-\lambda_{1} & \lambda_{1} & 0 & 0 & 0 & 0 & 0 \\
0 & -\lambda_{1} & \lambda_{1} & 0 & 0 & 0 & 0 \\
\hline 0 & 0 & -\lambda_{2} & \lambda_{2} & 0 & 0 & 0 \\
0 & 0 & 0 & -\lambda_{2} & \lambda_{2} & 0 & 0 \\
0 & 0 & 0 & 0 & -\lambda_{2} & \lambda_{2} & 0 \\
\hline 0 & 0 & 0 & 0 & 0 & -\lambda_{3} & \lambda_{3} \\
0 & 0 & 0 & 0 & 0 & 0 & -\lambda_{3}
\end{array}\right]
$$

The corresponding $\mathbf{S}^{\mathbf{0}}$ would have $\lambda_{3}$ as the last element, and all other elements 0 . We will omit the illustration of the estimated matrix with $\left(m_{1}, m_{2}, m_{3}\right)=(645,458,6)$. 


\subsubsection{Conditional Distribution of Sensor Observations}

The different patterns of the sensor measurement when the sow is in different states are what allow us to distinguish which state the sow is in. These conditional distributions are therefore an important part of the algorithm. The prediction algorithm was implemented to use four different sensor measures, namely, water observations, meanActivity, sdActivity and grid activity. Note that we use the log-transformation of these variables throughout the model. The conditional distributions also capture the diurnal rhythm of the sow hidden in the sensor observation in addition to the pre-parturition behaviour.

Video-Activity. The mean levels of the meanActivity and sdActivity were plotted against the time of a day, 0-24 hours, as shown in Figure 6. The lines indicating state-1, state-2 and state-3 correspond to Before Nest-Building, Nest-Building and Resting states. The mean levels were estimated with the SDs $\sigma^{(A m)}=0.8$ and $\sigma^{(A s d)}=0.9$. The peaks in the curves for state- 1 suggest the pronounced diurnal variation and the corresponding time of the day matches with the managemental records. In state- 2 the mean level reflects the highly active nest building behaviour of the sow. The pattern of the meanActivity and sdActivity is more or less identical.

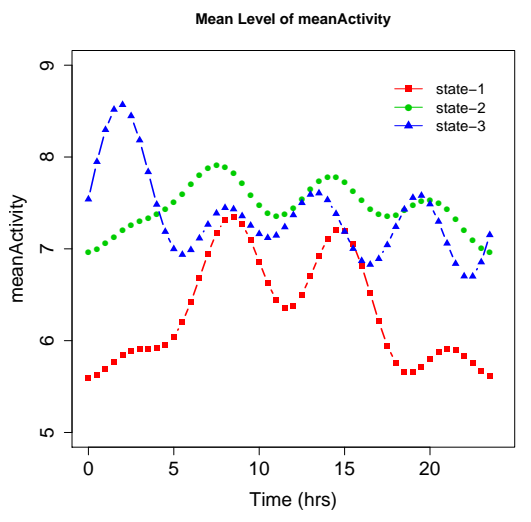

(a) meanActivity

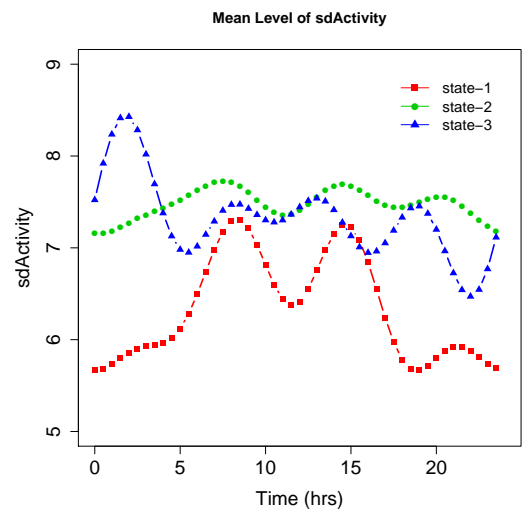

(b) sdActivity

Figure 6: Mean level of meanActivity and sdActivity over a day in three states of a sow; state-1 (Before Nest-Building) and state-2 (Nest-Building) and state-3 (Resting).

Grid Activity. The mean level of the grid activity was estimated with $\mathrm{SD}, \sigma^{(g)}=1.64$. The plot of $\mu^{(g)}$ over 24hours of a day is as shown in Figure 7. For state-1 the diurnal pattern is identical to that of Figure 6. In contrast to the video-activity, the mean grid-activity shows a marked difference between state- 2 and state-3. The reason is probably that the grid activity only measures the activity while standing up. If the sow moves while lying down, it will not show up in the grid measurements.

Water Consumption. The conditional distribution for water consumption data was modelled with three mixture components in the states Before Nest-Building and Nest-Building. The mean level of the components of Before Nest-Building state was estimated to be $\mu_{1}^{(w)}=[7.07,2.09,0]$ with the residual standard deviation $\sigma_{1}{ }^{(w)}=[0.96,1.14,0.01]$. That is, the Component-1 corresponded to the most drinking per drinking episode, Component-2 may be a kind of redirected behaviour, e.g. playing with the drinking nipple, and Component-3 to almost no drinking activity. The corresponding mixing probabilities varied throughout the day and are shown in Figure 8(a) (the x-axis of the plot denotes the 0-24 hours of a day). Early morning and mid-night, the water consumption was very low compared to that during the day time 


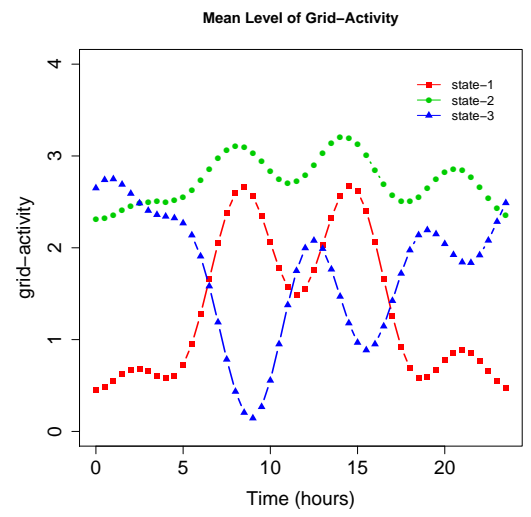

Figure 7: Mean level grid-activity over a day in three states of a sow: state-1 (Before Nest-Building), state-2 (Nest-Building) and state-3 (Resting).

(Component-3). Also, as the other measures the model captures the feeding times by showing large probability of drinking at around hour 8 and 15 of the day (the peaks for Component-1). Apart from this lower and higher level of water consumption, the sow has also intended to consume some water during the night/day time with the very low probability as denoted by the dots for Component- 2 . The estimates for mean level of water consumption for each component over a day for Nest-Building state were similar and are $\mu_{2}^{(w)}=[7.14,3.61,0.001]$ with residual standard deviation $\sigma_{2}{ }^{(w)}=[0.74,2.0,0.02]$. But as the plot of probabilities for the Nest-Building state, Figure 8(b), shows, there is a notable change in the consumption pattern. The plot shows more water activity even at the night time (after hour 20 and before hour 4)(Component-1), indicating a clear link between nest building activity and water intake.

Since the Resting state has very short duration we consider only one component for the water consumption with mean level estimated to be $\mu_{3}^{(w)}=0.49\left(\sigma_{3}{ }^{(w)}=1.61\right)$.

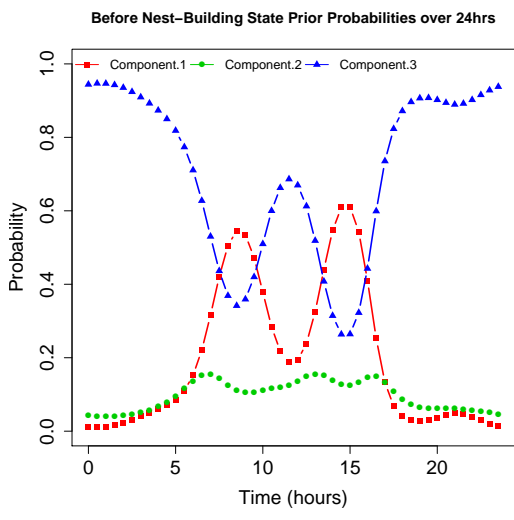

(a) Before Nest-Building

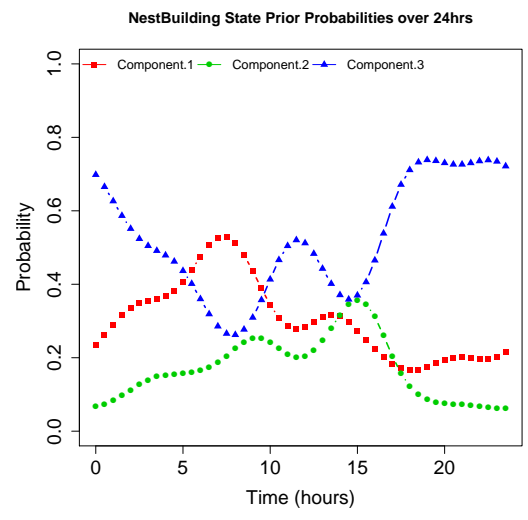

(b) Nest-Building

Figure 8: The mixture probabilities for water consumption over a day for the components of Before Nest-Building and Nest Building states. The consumption behaviour was classified into components: Component-1 to 3 correspond to most-drinking to no-drinking activities. Furthermore, $\operatorname{Pr}($ Component -1$)+\operatorname{Pr}($ Component -2$)+\operatorname{Pr}($ Component -3$)=1$, at a given time. 


\subsection{Validation of Warning Strategy}

In the present paper, the prediction algorithm was evaluated using the prediction in two different kind of simple and heuristic warning strategies. The first is based on the expected time to farrowing,

$$
\mathbb{E}[T]_{t+\delta}=-\boldsymbol{\alpha}_{t+\delta} \mathbf{S}^{-1} \mathbf{1}
$$

as in (3). This is inspired by the use of prediction for the climate regulation system that requires activation of floor heating prior to farrowing, early enough to ensure the desired level of floor temperature at the start of the farrowing.

The second was inspired by the management surveillance case, where it is natural to concentrate efforts on the sows that are most likely to farrow within a given time-period $x$. Thus we calculate the probability of farrowing in the next $x$ hours as

$$
\left\{\left(\bar{\alpha}_{x}\right)_{F}\right\}_{t+\delta}=1-\boldsymbol{\alpha}_{t+\delta} \mathbf{e}^{\mathbf{S} x} \mathbf{1}
$$

as in (1).

Each of these strategies were illustrated in sec. 5 by plotting the respective statistic value over the time of prediction. A warning or alarm was raised if the predicted statistic value is less than the pre-chosen threshold value and are indicated by a dot over the prediction line. The evaluation was made for different scenarios. For each of the scenarios, different success criteria was measured.

Since the alarms are time dependent, the types of alarms were defined based on the "alarm or warning period". We illustrate the warning periods and their types using the schematic diagrams in Figure 9. The curve connecting the points O-A-B-C-E-D forms a prediction curve. When the predicted time to farrowing drops below the threshold a warnings is raised. Thus, warnings were raised in between the points $\mathrm{A}$ and $\mathrm{B}$ (Curve-AB) as well as between the points C, E and D (Curve-CED). Curve-AB and Curve-CED are therefore, two warning periods. A true warning period constitutes coherent warning periods that continues until the farrowing is observed (for example, Curve-CED). If the warning period is interrupted before the farrowing or the warnings are canceled by the later predictions, it is false warning period (for example, Curve-AB). Thus the warning algorithm tells the farmer when a warning alarm is no longer valid.

Each sow can have only one true warning period. The number of sows with true warning periods are counted. For the true warnings, we calculate the mean, standard deviation, maximum and the third quartile $\left(Q_{3}\right)$ of the warning duration for all the sows. However, there can be more than one incoherent false warning periods for one sow. For example, if Curve-OABCEFG of Figure 9 is the prediction curve for a sow, Curve-AB and Curve-CEF are false-warning periods. For the false warnings we calculate the total duration for each sow and then the mean of these durations over all the sows. The latter is called the Error. In the floor heating case, it would correspond to how long time the heat was turned on unnecessarily. The results are presented in the Table 2. For one of the scenario, we also present the table of number of sows, number of false warning periods and the mean duration of period.

In the present paper, the first strategy was tested for the threshold value 12 hours. That is, an alarm was raised if the expected time to farrowing was less than 12 hours. For the same strategy and scenario, the performance of the prediction algorithm was evaluated for different combination of water, videoactivity and grid-activity sensors; this include, \{water and video-activity\}, individual performances of water and video-activity, $\{$ water, video-activity and grid-activity $\},\{$ water and grid-activity $\}$, $\{$ video and grid-activity $\}$. Whenever meanActivity was calculated, sdActivity was also calculated. Therefore, we include both the measures if the combination consists video-activity. 
For the combination of water and video-activity sensors, the performance of the algorithm was also compared by changing the threshold to 2 and 6 hours. Evaluating the algorithm and the strategy for the reduced thresholds is also necessary for the applications like climate controlling (for example, when a higher input energy was supplied) and managemental surveillance.

The second strategy was tested by calculating the probability of farrowing in the next $x=12$ hours. The warning results were compared by varying the probability threshold from 0.4 to 0.6 .

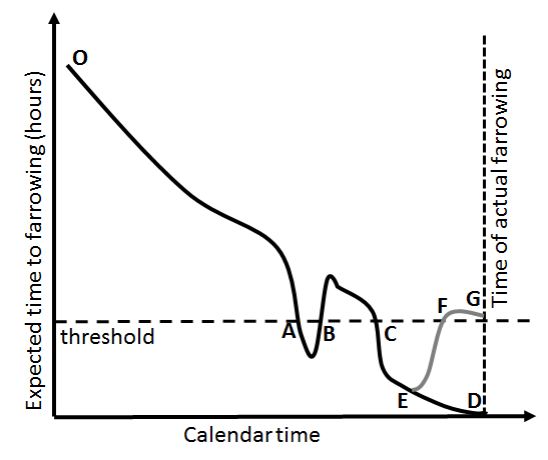

Figure 9: Schematic diagrams of prediction curve and the alarms types. Curve-OABCED is the prediction curve for a sow. An alarm or warning is raised if the prediction falls below the threshold line. Curve-AB and Curve-CED are warning periods. Since Curve-AB is interrupted, it is false warning period; whereas Curve-CED continues till the actual farrowing and hence, it is a true warning period. A sow can have only one true warning period; but may have more than one incoherent false warning periods such as Curve-AB and Curve-CEF of the prediction Curve-OABCEFG.

\subsection{Computational Environment}

The prediction algorithm was implemented in the statistical computational environment $\mathrm{R}$ (R Development Core Team, 2010). Various functions supporting the algorithm were written. These functions have been collected into a package compatible with $\mathrm{R}$.

\section{Results}

\subsection{Illustration of use of algorithm for individual sows}

The output of the prediction algorithm compared with the actual time of onset of farrowing for one sow is illustrated in Figure 10(a). The algorithm has used information from water and video-activity sensors. The time-axis starts from the time the sow was inserted into the farrowing pen, and the horizontal dashed line is drawn to indicate the threshold for the warning strategy. The vertical line indicates the actual day of farrowing of that sow. The expected time to farrowing was calculated at every prediction time step and are plotted against the time-axis. The expected time to farrowing decreased linearly as long as the observations did not revise the probability distribution over phases, because the most likely state was the Before Nest-Building. As the time has passed, other states become more likely, and the prediction began to change. A small drop on January $13^{\text {th }}$ was the clear indication of the sow in the Nest-Building state. Visual inspection of the video recordings for the sow has confirmed the nest-building on January 
$14^{t h}$. The plot has also showed a sudden drop in the expected time when the sow was very close to farrowing and continued to decrease over time, indicating that the sow was approaching the farrowing state.

For this case, the warning threshold was set to 12 hours. However, the true warnings were raised 13.3 hours before the actual farrowing. If the algorithm uses only video-activity data during the prediction, the true warnings were raised 13.8 hours before the actual farrowing (see Figure 10(b)). On the other hand, if only water consumption data was used in the algorithm, there would not have been any warnings (see Figure 10(c)); indeed, if we assume that the farmer routinely visits the herd on every day at 10:30 am then there would have been a delay of 6.7 hours in knowing the farrowing.

The algorithm may also give false warnings, such as in Figure 11. In the example Figure 11(a), around July 2, some of the observations led to a marked drop resulting in false warnings; 2 times before farrowing. These false warnings were there for 2 and 5 hours respectively. The prediction was revised later on and hence, the warnings were retracted. On July 5 , the observations clearly indicated a transition to the Nest-Building state (confirmed by the visual inspection of the video recordings), and the expected time to farrowing dropped below 12 hours. Therefore, a warning was raised 8.3 hours before farrowing and are indicated by the dots in the plot. However, in Figure 11(b), the sow has only false warnings and no alarm during the farrowing.

The strategy of probability of farrowing in the next 12 hours is illustrated in Figure 12. The horizontal lines indicate different threshold points, from 0.4 to 0.6 . The first alarm was produced on January $14^{t h}$, almost 14 hours before farrowing. The probability curve has sharpened as the farrowing approaches.

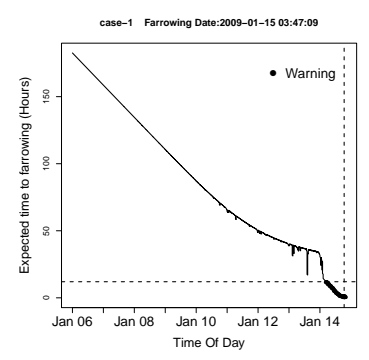

(a) water and video-activity

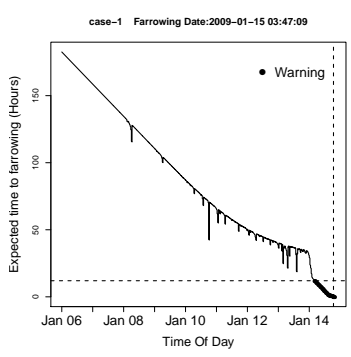

(b) video-activity

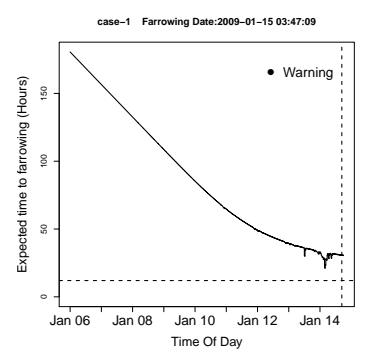

(c) water

Figure 10: Prediction curves for one sow with different combination of water and video-activity sensors for the strategy expected time to farrowing with threshold 12 hours.

\subsection{Validation of algorithm for different sensor combinations and heuristic strategies}

The sample size, percentage of true warnings, mean, standard deviation, maximum and third quartiles of true warnings and the error due to false warnings for different warning strategies, threshold scenarios and combination of sensors are presented in Table 2. For the combination of water and video-activity, with threshold 12 hours for the expected time to farrowing strategy, 34 of the 35 sows (97\%) gave satisfactory warnings with mean 11.5 hours ( $\mathrm{SD}=4.6$ hours), with the mean error of 0.7 hours. 29 of 35 sows (83\%) have resulted in only true warning periods. 2 of 35 sows had 2 periods of false warning and 4 of 35 had only one false warning period with mean error 3.75 and 4.6 hours, respectively (see Table 3 ).

Using only water measures, the algorithm gave only 8 true-warnings out of 38 (21\%) with mean of 11.7 hours ( $\mathrm{SD}=2.2$ hours). However the error was increased to 3.4 hours. The algorithm which has used 


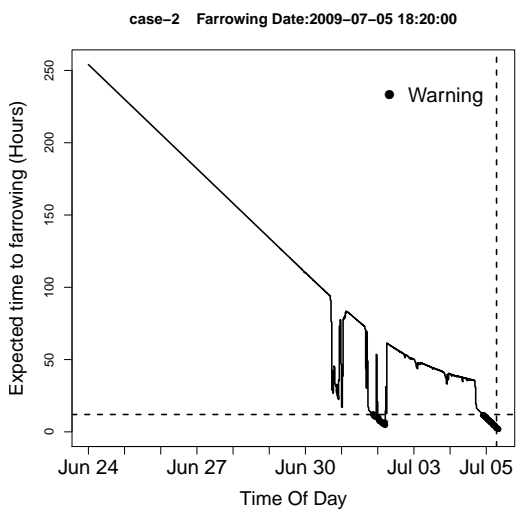

(a) case-2

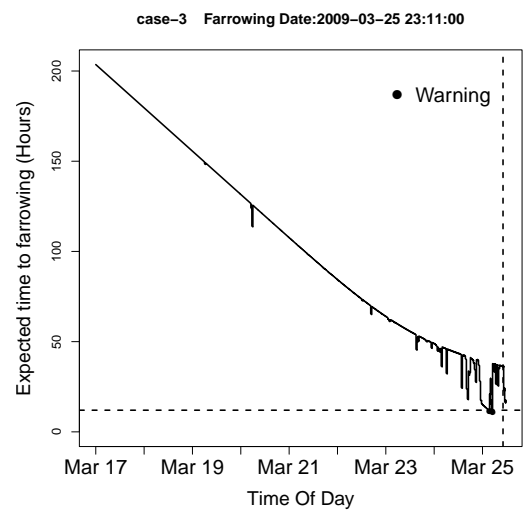

(b) case-3

Figure 11: Illustration of false warning period with (case-2) and without (case-3) a true-warning period.

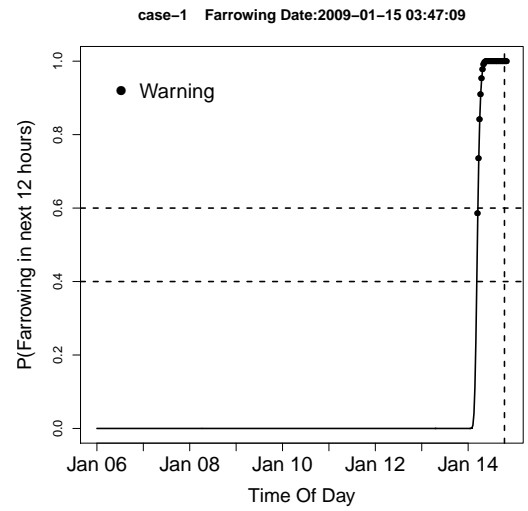

Figure 12: Prediction curve with probability of farrowing in next 12 hours against time of prediction. The horizontal lines correspond to the threshold limits 0.4 and 0.6. The prediction curve sharpened as farrowing approaches. 
Table 2: Comparison of prediction algorithm for different combination of sensors and for different warning-strategies. The sensors are abbreviated as $\mathrm{W}$ : water consumption, A: video-activity measures, G: grid-activity measures. Warning strategies are ETF: Expected time to farrowing based strategy, PF: the strategy being Probability of farrowing in next 12 hours. Error: mean duration of false warning period (hours). $Q_{3}$ : third quartile of true-warning period. Threshold values for ETF strategy are in hours.

\begin{tabular}{|c|c|c|c|c|c|c|c|c|c|}
\hline \multirow{3}{*}{ Strategy } & \multirow[b]{2}{*}{ Sensor } & \multirow{3}{*}{$\begin{array}{c}\text { Threshold } \\
12\end{array}$} & \multirow{3}{*}{$\begin{array}{c}\begin{array}{c}\text { Sample } \\
\text { Size }\end{array} \\
35\end{array}$} & \multirow{3}{*}{$\begin{array}{c}\begin{array}{c}\text { True } \\
\text { warnings } \\
\%\end{array} \\
97\end{array}$} & \multicolumn{4}{|c|}{ True Warning Period } & \multirow[b]{2}{*}{$\begin{array}{r}\text { Error } \\
\text { (hours) }\end{array}$} \\
\hline & & & & & \multicolumn{2}{|c|}{$\begin{array}{l}\text { (hours) } \\
\end{array}$} & \multicolumn{2}{|c|}{$\underset{\text { (hours) }}{\operatorname{Max}} Q_{3}$} & \\
\hline & & & & & 11.5 & 4.6 & 22.6 & 14.6 & 0.7 \\
\hline \multirow[t]{2}{*}{ ETF } & WA & 6 & 35 & 83 & 6.9 & 3.4 & 16.6 & 8.7 & 0.1 \\
\hline & & 2 & 35 & 60 & 4.2 & 2.6 & 12.6 & 5.1 & 0.1 \\
\hline \multirow{2}{*}{ ETF } & W & \multirow{2}{*}{12} & 38 & 21 & 11.7 & 2.2 & 13.9 & 13.2 & 3.4 \\
\hline & A & & 55 & 98 & 14.4 & 12.5 & 93.9 & 15.0 & 1.6 \\
\hline $\mathrm{PF}$ & WA & 0.4 & 35 & 97 & 11.7 & 4.5 & 22.6 & 14.7 & 1.1 \\
\hline \multirow{3}{*}{ ETF } & WAG & \multirow{3}{*}{12} & 34 & 97 & 11.6 & 4.8 & 22.6 & 14.4 & 0.8 \\
\hline & WG & & 36 & 67 & 12.2 & 5.6 & 34.8 & 13.3 & 3.8 \\
\hline & $\mathrm{AG}$ & & 37 & 100 & 13.5 & 7.3 & 40.4 & 15.9 & 3.1 \\
\hline
\end{tabular}

Table 3: Count and mean duration of false warning periods for the scenario Strategy: expected time to farrowing (ETF), Sensor: Water and Video-Activity, Threshold: 12hours

\begin{tabular}{llll}
\hline No. of periods with false warnings & 0 & 1 & 2 \\
\hline No. of sows & 29 & 4 & 2 \\
Mean duration (hours) & - & 4.6 & 3.75 \\
\hline
\end{tabular}


only video-activity information (meanActivity and sdActivity) gave 98\% true-warnings and are penalized by the bias in the mean of 14.4 hours and the increased SD of 12.5 hours. The combination of water and video-activity sensors with grid-activity in the prediction algorithm did not improve the prediction results. When used with water or video-activity, the mean time of true-warnings did not improve much. However, with the water, the grid-activity sensor increased the number of true-warnings to $67 \%$ with $\mathrm{SD}=5.6$ hours and error 3.8 hours. Using the grid-activity sensor with the video-activity sensor decreased in the SD warning time from 12.5 hours to 7.3 hours.

If the threshold was decreased to 6 hours for water and video-activity combination, only 29 of 35 sows gave true-warnings with mean warning time 6.9 hours ( $\mathrm{SD}=3.4$ hours) and an error 0.1 hours. If the threshold was further reduced to 2 hours, only 21 sows gave true-warnings with a biased mean warning time 4.2 hours ( $\mathrm{SD}=2.6$ hours). The prediction algorithm had in general low bias. The expected mean time to farrowing used as threshold was close to the observed mean value.

For the strategy of probability of farrowing in the next 12 hours, for the threshold 0.4 , the algorithm predicted 36 true-warnings out of 38 with mean warning time of 11.4 hours ( $\mathrm{SD}=4.2$ hours). Since the probability curve has a steep rise as farrowing approaches, results of other thresholds are identical to that for 0.4 .

\section{Discussion and Conclusions}

The method applied in our study has originated in life-time or time to failure studies and therefore, the algorithm presented is directly focused on predicting the probability distribution of remaining time to farrowing (which corresponds to the failure time in the other studies). The biological knowledge of change in behavioural states of the sow in the farrowing pen gave a good modelling framework for handling different patterns in the sensor measures. One of the characteristics of the sensor measurements was a marked diurnal variation. This has allowed a combination of two time scales in the prediction model; the time of the day and the time since mating. The diurnal variation and the latent state/phase allowed us to treat the different sensor measurements as independent given the state. Thus, we could easily adapt the complexity of the modelling to each different sensor observations. Moreover, this prediction algorithm gives a framework to integrate information from different sensor types for the prediction; with an identical model and model-parameters, simply by treating some of the observations as missing values. The prediction algorithm performed best using both activity and water consumption, although the individual performance of the information from the water sensor was not promising. Thus the combination of these sensors is recommended. Furthermore, because the prediction algorithm can update with values from any sensor, the farmer may choose a suitable sensor for his herd depending on the resources available. The prediction algorithm seems to be unbiased with respect to the threshold level, the observed mean time to farrowing in the validating corresponds to the expected time to farrowing used as threshold. For management surveillance the probability based strategy which can rank the sows according to the probability of farrowing within, say, 12 hours, may also be of interest. Since the earliest start of the Nest-Building state is later than 24 hours before farrowing, it is not advisable to use the probability of farrowing within 24 hours as a prediction measure.

In our study, the alarms were evaluated on the time of the alarm and the time of farrowing. This is similar to an ordinary fire alarm system. According to our evaluation criteria, a true alarm period should continue until the farrowing is observed; otherwise, it is sensible to end the alarm period if the new evidence indicates so and such a period should be treated as a false warning (as illustrated in Figure 11(a)). After the false alarm period, the algorithm should revise its knowledge and continue the prediction. From 
the results, it is also clear that new sensor observation may revise the expected time to farrowing. The intended use of the prediction algorithm for activation of a floor heating system made it clear that a relevant warning system should give warning in time for the floor to heat up and it also helped in defining relevant measures of success used in this paper, as described in section 4.2. There may exist early periods where a warning is triggered, because the expected duration falls below the threshold, but later the expected duration increases above the threshold. From the heating point of view the heat will be turned on, but energy may be wasted because the floor temperature will return to room temperature before farrowing. Only when the warning period extends until start of farrowing, the energy will be used fully, leading to a true warning.

The discussion above illustrates the need for a clearer definition of how to treat the problems of sequential observations, that may lead to alarms. One way is to make a more direct specification of the cost and benefits that may arise from the alarms. The phase-type formulation makes it possible to set up a decision support system or automatic heat regulation system based on the methods described in Lovejoy (1991); Aberdeen (2003). We have implemented such a system in chapter 4 of Aparna (2013) for the floor-heat regulation in farrowing pen. This system takes the sequential nature of the decision making into account and includes the expected outcome of future observations. This system allows a systematic approach to precision of prediction, false versus true alarms, and multiple alarms, in contrast to the different heuristic strategies used in the present paper.

Our algorithm performed satisfactorily based on the evaluation criteria (Table 2) and a cost-benefit analysis has confirmed that the use of the algorithm and the sensors is expected to be cost effective, after further product development (Jørgensen et al., 2010). As demonstrated in chapter 4 of Aparna (2013) the algorithm may also be used directly for calculating optimal heating decisions.

Similar system could be made with respect to warning to improve the efficiency of surveillance of farrowings, as seems to be the intention of the development of algorithms in Cornou and LundbyeChristensen (2012).

Unfortunately it is difficult to compare our results with the study of Cornou and Lundbye-Christensen (2012), the only study with similar aim of sending warnings about farrowings, as mentioned in Section 1; but they use a different methodology, and a different approach for evaluation. In both the methods applied in Cornou and Lundbye-Christensen (2012), the criteria for alarm is related to the detection of change in activity pattern and were evaluated by noting whether any alarm is raised within the last 24 hours before farrowing.

Furthermore, the prediction algorithm presented here can be used to predict or detect the beginning of Nest-Building state. Prediction may be done by considering the first phase of the Nest-Building state as an absorbing phase. Both prediction and detection of the Nest-Building state may be helpful, say, in providing the nest building materials to the sow as soon as possible after the start of nest building behaviour.

Another issue could be to use a finer time interval for the observations than the half an hour interval. However, this may lead to a model with autocorrelation of the sensor observations, and if this is included, it would increase the the model complexity significantly.

The assumption of conditional independence of the sensor measurements may also be questioned. The primary reason for this assumption is to keep the model parsimonious. Without this assumption, modelling the interaction with diurnal rhythm will further increase the complexity of the model. However, if there is an effect of dependency and if it is not included in the model then the model may overestimate 
the precision in our knowledge of the phase number after a series of observations. This would lead to poorer predictions of farrowing time. In that sense, the value of our model is at least not overestimated. Pooling the data into half hour intervals may reduce the dependency between the measurements of different sensors. These arguments are also supported by our results in Table 2; results show that different sensors contribute different information about the behaviour. If not, the combination of sensors would not be expected to give better prediction results.

The computational complexity of the algorithm is mainly related to the number of phases (approximately 1000), where the transition matrix will be of size $1000 \times 1000$. This matrix is multiplied to the state vector every half hour for each sow. This should not give any problems on standard modern computers as used on farm. The algorithm may be revised to use some other information such as farmer's routine visit to the herd which in turn may be a better evidence about the farrowing. The success of the algorithm to adopt warning strategies makes the phase-type approach promising in other applications exploiting sensor information in precision livestock agriculture. In fact O'Connell et al. (2011) has applied a similar semi-Markov process to the problem of oestrus detection in dairy cows. Prediction of possible disease outbreaks and outbreak of behavioral problems such as tail-biting are other obvious candidates.

\section{Acknowledgements}

The study is part of the project 'The Intelligent Farrowing Pen', financed by the Danish National Advanced Technology Foundation. 
Aberdeen, D., 2003. A (revised) survey of approximate methods for solving partially observable markov decision processes. National ICT Australia, Canberra, Australia, Tech. Rep.

Andersen, I., Haukvik, I., Bøe, K., 2009. Drying and warming immediately after birth may reduce piglet mortality in loose-housed sows. Animal 3 (04), 592-597.

Andersen, I. L., Tajet, G. M., Haukvik, I. A., Kongsrud, S., Bøe, K. E., 2007. Relationship between postnatal piglet mortality, environmental factors and management around farrowing in herds with loosehoused, lactating sows. Acta Agriculturae Scandinavica, Section A - Animal Science 57 (1), 38-45. URL http: / / www .tandfonline.com/doi/abs/10.1080/09064700601159626

Aparna, U., 2013. Methods for sensor based farrowing prediction and floor-heat regulation: The intelligent farrowing pen. Ph.D. thesis, Graduate School of Science and Technology, Aarhus University, under evaluation.

Asmussen, S., Nerman, O., Olsson, M., 1996. Fitting phase-type distributions via the em algorithm. Scandinavian Journal of Statistics 23 (4), 419-441.

URL http: / /www.jstor.org/stable/4616418

Baxter, E., Lawrence, A., Edwards, S., 2011. Alternative farrowing accommodation: welfare and economic aspects of existing farrowing and lactation systems for pigs. Animal 6 (1), 96-117.

URL http://journals.cambridge.org/download.php?file=2FANM2FANM6_

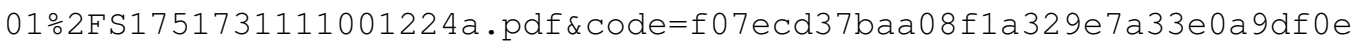

Baxter, S., 1984. Intensive pig production: environmental management and design. Granada Publishing Ltd.

Bernstein, D., 2009. Matrix mathematics: theory, facts, and formulas. Princeton University Press.

Bressers, H., te Brake, J., Jansen, M., Nijenhuis, P., Noordhuizen, J., 1994. Monitoring individual sows - radiotelemetrically recorded ear base temperature changes around farrowing. Livestock Production Science 37, 353-361.

Castrén, H., Algers, B., de Passillé, A.-M., Rushen, J., Uvnäs-Moberg, K., 1993. Preparturient variation in progesterone, prolactin, oxytocin and somatostatin in relation to nest building in sows. Applied Animal Behaviour Science 38 (2), 91 - 102.

URL http://www.sciencedirect.com/science/article/pii/ $016815919390059 X$

Cornou, C., Lundbye-Christensen, S., 2012. Modeling of sows diurnal activity pattern and detection of parturition using acceleration measurements. Computers and Electronics in Agriculture 80 (0), 97 104.

URL

http://www.sciencedirect.com/science/article/pii/

S0168169911002481

Cox, D. R., 1955. A use of complex probabilities in the theory of stochastic processes. Mathematical Proceedings of the Cambridge Philosophical Society 51 (02), 313-319.

URL http://journals.cambridge.org/action/displayAbstract?frompage= online\&aid=2046444\&fulltext Type=RA\&fileId=S0305004100030231

Dayanik, S., Goulding, C., 2009. Sequential detection and identification of a change in the distribution of a markov-modulated random sequence. Information Theory, IEEE Transactions on 55 (7), 3323-3345. 
Erez, B., Hartsock, T., 1990. A microcomputer-photocell to monitor periparturient activity of sows and transfer data to remote location. Journal of Animal Science 68, 88-94.

James, G., Witten, D., Hastie, T., Tibshirani, R., 2013. An introduction to statistical learning. Springer.

Jensen, P., 1989. Nest site choice and nest building of free-ranging domestic pigs due to farrow. Applied Animal Behaviour Science 22 (1), $13-21$.

URL http://www.sciencedirect.com/science/article/pii/

0168159189900762

Jørgensen, E., U, A., Jensen, K. M., Sørensen, L. C., Andersen, H. M., 2010. Working paper, cost -benefit. intelligent farrowing pen. version 2. october 29, 2010. appendix 3 in "go/no-go milepælsrapport. den intelligente faresti. højteknologifonden. [1. nov 2010. Tech. rep., Dept. of Genetics and Biotechnology, Aarhus University", internal report.

URL http://gbi.agrsci.dk/ ejo/publications/preprints/IFCosts2010.pdf

Lovejoy, W., 1991. A survey of algorithmic methods for partially observed markov decision processes. Annals of Operations Research 28 (1), 47-65.

URL http://dx.doi.org/10.1007/BF02055574

Madsen, T. N., Andersen, S., Kristensen, A. R., 2005. Modelling the drinking patterns of young pigs using a state space model. Computers and Electronics in Agriculture 48 (1), 39-61.

URL http://www.sciencedirect.com/science/article/B6T5M-4FR8PFN-1/2/ 3c273683415228e11e67cb634923edal

Madsen, T. N., Kristensen, A. R., 2005. A model for monitoring the condition of young pigs by their drinking behaviour. Computers and Electronics in Agriculture 48 (2), 138-154.

URL http://wWw.sciencedirect.com/science/article/B6T5M-4FVH3X7-1/2/ d5a42c8fad8d42da2417d20a33932fe0

Malmkvist, J., Pedersen, L., Damgaard, B., Thodberg, K., Jorgensen, E., Labouriau, R., 2006. Does floor heating around parturition affect the vitality of piglets born to loose housed sows? Applied Animal Behaviour Science 99 (1-2), 88-105.

Malmkvist, J., Pedersen, L., Sund Kammersgaard, T., Jørgensen, E., 2012. Influence of thermal environment on sows around farrowing and during the lactation period. Journal of Animal Science 90 (9), 3186-3199.

URL http: / / www. journalofanimalscience.org/content/90/9/3186

Neuts, M. F., 1975. Probability distributions of phase type. Liber Amicorum Professor Emeritus H. Florin, University of Louvain, Belgium, 173-206.

Neuts, M. F., 1978. Renewal processes of phase type. Naval Research Logistics Quarterly 25 (3), 445454.

URL http://dx.doi.org/10.1002/nav.3800250307

O’Connell, J., Tøgersen, F. A., Friggens, N. C., Løvendahl, P., Højsgaard, S., 2011. Combining cattle activity and progesterone measurements using hidden semi-markov models. Journal of Agricultural, Biological, and Environmental Statistics 16 (1), 1-16.

URL http: / /dx.doi.org/10.1007/s13253-010-0033-7

Oliviero, C., Pastell, M., Heinonen, M., Heikkonen, J., Valros, A., Ahokas, J., Vainio, O., Peltoniemi, O. A., 2008. Using movement sensors to detect the onset of farrowing. Biosystems Engineering 
100 (2), 281-285.

URL http://WWW.sciencedirect.com/science/article/B6WXV-4SG01GW-2/2/ 543577118 e1c584cce676ba4ed0520ff

R Development Core Team, 2010. R: A Language and Environment for Statistical Computing. R Foundation for Statistical Computing, Vienna, Austria, ISBN 3-900051-07-0.

URL http: / / www.R-project.org/

Thodberg, K., Jensen, K. H., Herskin, M. S., 2002. Nest building and farrowing in sows: relation to the reaction pattern during stress, farrowing environment and experience. Applied Animal Behaviour Science 77 (1), 21 - 42.

URL http://wWw.sciencedirect.com/science/article/B6T48-45F8Y1Y-1/2/ 1 b 78 d 10 ca 4 a 4 b 3 c 80 c 45 dee 35 d 6 a 4740

Vinther, J., 2013. Landsgennemsnit for produktivitet i svineproduktionen 2012 (national productivity averages in pig production 2012). Videnscenter for Svineproduktion 1314, 1-5.

Wallgren, T., 2013. Variation in piglet mortality between and within satellites in a sow pool. Master's thesis, SLU, Dept. of Animal Breeding and Genetics.

West, M., Harrison, J., 1997. Bayesian forecasting and dynamic models, 2nd Edition. Springer-Verlag, New York.

White, K., Anderson, D., Bate, L., 1996. Increasing piglet survival through an improved farrowing management protocol. Canadian Journal of Animal Science 76 (4), 491-495.

Wischner, D., Kemper, N., Krieter, J., 2009. Nest-building behaviour in sows and consequences for pig husbandry. Livestock Science 124 (1-3), 1-8.

URL

http://www.sciencedirect.com/science/article/pii/

S1871141309000328 